\section{International Scientific Journal Theoretical \& Applied Science}

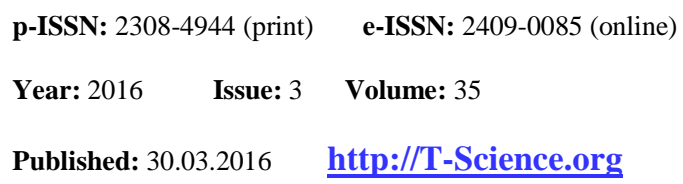

SECTION 20. Medicine.
Gulshad Gafurkhanovna Sarabitdinova

Master's Student in Medical Sciences International Kazakh-Turkish University by name Yassavi, Kazakhstan sharabitdinova80@inbox.ru

Assel Dauletbayevna Sadykova Ph.D. Student in Medical Sciences International Kazakh-Turkish University by name Yassavi, Kazakhstan aselyasadykova@gmail.com

Dilorom Kamilzhanovna Ibragimova

Master's Student in Medical Sciences International Kazakh-Turkish University by name Yassavi, Kazakhstan dil1902@mail.ru

Saltanat Seilbekovna Askarova Candidate of Medical Sciences Lecturer International Kazakh-Turkish University by name Yassavi, Kazakhstan askarovas@mail.ru

Gulnaz Orazbekovna Nuskabayeva a.e. associate professor Candidate of Medical Sciences Head of Department International Kazakh-Turkish University by name Yassavi, Kazakhstan g_nuskabaeva@mail.ru

Zhanar Sailaubekovna Shalkharova MD, Vice-president "GI Educational and Healthcare Holding" zhanar.shalkharova@gmail.com

Zhanat Nagmetovna Shalkharova Professor, MD, Lecturer International Kazakh-Turkish University by name Yassavi, Kazakhstan zhanatsh@mail.ru

\title{
THE ROLE OF THE COMBINATIONS OF GENES POLYMORPHISMS OF THE BETA 1- AND BETA 3 ADRENERGIC RECEPTORS IN A HEART VASCULAR DISEASES AND DIABETES MELLITUS TYPE 2
}

Abstract: In this article we studied clinical metabolic determinants depending on the genes polymorphism combinations of beta 1 - and beta 3 adrenergic receptors.

Key words: gene polymorphism, beta 1 - and beta 3 adrenergic receptors, clinical and metabolic determinants. Language: English

Citation: Sharabitdinova GG, Sadykova AD, Ibragimova DK, Askarova SS, Nuskabaeva GO, Shalkharova ZS, Shalkharova ZN (2016) THE ROLE OF THE COMBINATIONS OF GENES POLYMORPHISMS OF THE BETA 1- AND BETA 3 ADRENERGIC RECEPTORS IN A HEART VASCULAR DISEASES AND DIABETES MELLITUS TYPE 2. ISJ Theoretical \& Applied Science, 03 (35): 159-164.

Soi: http://s-o-i.org/1.1/TAS-03-35-27 Doi: crossef http://dx.doi.org/10.15863/TAS.2016.03.35.27 
BACKGROUND: The main role in developing of heart vascular diseases (HVD) and diabetes mellitus type 2 acts genes [1]. Many studies have discussed genes which are responsible for evolution HVD and diabetes mellitus type 2 [2-4]. Similar researches were performed in Kazakhstan [5-8].

Nowadays is determined that genes polymorphism combinations have impact on the development of HVD and diabetes mellitus type 2 . Researchers from Chile studying the link between the gene polymorphism combinations of beta 1 (ADRB1) and beta 3 adrenergic receptors (ADRB3) with sudden death, determined that people with ADRB1Arg389Gly and ADRB2 Gln27Glu gene polymorphisms combinations have a high risk of sudden death [9]. The aim of the Polish scientists was to study the association of polymorphisms gene between beta 1 adrenergic receptors (Ser49Gly, Arg389Gly) and beta 2 adrenergic receptors (Arg16Gly, Gln27Glu, Thr164Ile) with idiopathic dilated cardiomyopathy. The results showing that, beta-adrenergic receptor polymorphisms did not seem to play a significant role in IDCM in the Polish population. [10]. There was also determined the association of gene polymorphism combinations of beta 1 and beta 3 adrenergic receptors Arg389Gly and Trp64Arg with the evaluating of metabolic changes of the patients with diabetes mellitus type 2 and metabolic syndrome [11].

AIM: To study combinations of the genes polymorphism of beta 1 and beta 3 adrenergic receptors depending on clinical metabolic and death rates.

DESIGN: We conducted this as a retrospective cohort study.

MATERIAL AND METHODS: We took the initial material of Shalkharova Zh.S., 2003 [12], Nuskabaeva G.O., 2003[7], Askarova S.S., 2003 [13] and were able to examine 130 patients with the gene polymorphism combinations of beta 1 and beta 3 adrenergic receptors, which were included further in our research. From whole 130 people 21 were deaths.
We investigated 109 alive patients, whose mean age was $57,42 \pm 13,34$. All patients, which were included in our study gave their agreement to participate in it. They were divided into 5 groups, depending on the genes of polymorphism combinations of beta 1 and beta 3 adrenergic receptors:

1. Arg/Arg+Trg/Trg - 66 volunteers $-50,8 \%$

2. Arg/Arg+Trg/Arg - 21 volunteers $-16,1 \%$

3. Arg/Gly+Trg/Trg - 30 volunteers $-23,1 \%$

4. Arg/Gly + Trg/Arg - 10 volunteers $-7,7 \%$

5. Arg/Arg+ Arg/Arg - 3 volunteers $-2,3 \%$

We made analysis by the next determinants:

1. Clinical antropometric: weight, height, waist (WC) and thigh circumstances, body mass index (BMI)

2. Biochemic analyses: glucose level of blood (norm is 3.89-5.6 $\mathrm{mmol} / \mathrm{l}$ ), total cholesterol (norm 3.3-5.0 mmol/l), HDL cholesterol (norm for men $\geq$ 1.0, for women $\geq 1.20 \mathrm{mmol} / \mathrm{l}$ ), triglycerides (norm $<$ $1.70 \mathrm{mmol} / \mathrm{l}$ ). LDL cholesterol was claimed by Friedewald W.T. [14]: LDL cholesterol= Total cholesterol - HDL cholesterol- Triglycerides/2,2.

Molecular genetic analysis of genes polymorphism combinations of beta 1 and beta 3 adrenergic receptors examined 12 years ago in a laboratory of human being genome by Scientific Research Institute of molecular biology and genetics of plants in the Republic of Uzbekistan under the direction of doctor of medicine, prof. Mukhamedov R.S. [13].

Statistical analysis was made using MS EXCEL, Biostat software.

RESULTS: By the results of our study from 130 volunteers 21 were dead by the time of the research (Table 1). In the group with the genes polymorphism combinations of beta 1 and beta 3 adrenergic receptors Arg/Arg+Trg/Trg died 14 respondents (21.2\%), Arg/Arg+Trg/Arg - 1 respondents $(4.8 \%), \quad$ Arg/Gly+Trg/Trg - 4 respondents (13.3\%), Arg/Gly+Trg/Arg - 2 respondents $(20 \%)$, consequently.

Table 1

Distribution of deaths by the genes polymorphism combinations of beta 1- and beta 3 adrenergic receptors.

\begin{tabular}{|l|l|l|l|l|l|l|l|l|}
\hline Genes polymorphism & \multicolumn{2}{l}{ Arg/Arg+ Trg/Trg } & \multicolumn{2}{l|}{ Arg/Arg+ Trg/Arg } & \multicolumn{2}{l|}{ Arg/Gly+ Trg/Trg } & \multicolumn{2}{|c|}{ Arg/Gly+ Trg/Arg } \\
\cline { 2 - 9 } $\begin{array}{l}\text { combinations of beta 1 } \\
\text { and beta 3 adrenergic } \\
\text { receptors }\end{array}$ & $\mathrm{n}$ & $\%$ & $\mathrm{~N}$ & $\%$ & $\mathrm{n}$ & $\%$ & $\mathrm{n}$ & $\%$ \\
\hline Total & 66 & $100 \%$ & 24 & $100 \%$ & 30 & $100 \%$ & 10 & $100 \%$ \\
\hline Death & 14 & $21,2 \%$ & 1 & $4,1 \%$ & 4 & $13,3 \%$ & 2 & $20,0 \%$ \\
\hline
\end{tabular}

Acute stroke was statistically significantly frequently founded by distributing the causes of death (42.1\%). And $12.1 \%$ of people with genes polymorphism combinations of beta 1 and beta 3 adrenergic receptors Arg/Arg+Trg/Trg died with acute stroke (Table 2). 


\begin{tabular}{|c|c|c|c|c|c|c|}
\hline Impact Factor: & $\begin{array}{l}\text { ISRA (India) } \\
\text { ISI (Dubai, UAF } \\
\text { GIF (Australia) } \\
\text { JIF }\end{array}$ & $\begin{array}{l}=1.344 \\
=0.829 \\
=0.564 \\
=1.500\end{array}$ & $\begin{array}{l}\text { SIS (USA) } \\
\text { PИНЦ (Russia) } \\
\text { ESJI (KZ) } \\
\text { SJIF (Morocco) }\end{array}$ & $\begin{array}{l}=0.912 \\
=0.179 \\
=1.042 \\
=2.031\end{array}$ & $\begin{array}{l}\text { ICV (Poland) } \\
\text { PIF (India) } \\
\text { IBI (India) }\end{array}$ & $\begin{array}{l}=6.630 \\
=1.940 \\
=4.260\end{array}$ \\
\hline
\end{tabular}

Table 2

Causes of death of patients with the genes polymorphism combinations of beta 1- and beta 3 adrenergic receptors.

\begin{tabular}{|l|l|l|l|l|l|l|l|l|}
\hline $\begin{array}{l}\text { Gene polymorphism } \\
\text { combinations of beta 1 } \\
\text { and beta 3 adrenergic } \\
\text { receptors }\end{array}$ & \multicolumn{2}{l|}{ Arg/Arg+ Trg/Trg } & \multicolumn{2}{l|}{ Arg/Arg+Trg/Arg } & \multicolumn{2}{l|}{ Arg/Gly+Trg/Trg } & \multicolumn{2}{l|}{ Arg/Gly+ Trg/Arg } \\
\hline & $\mathrm{n}$ & $\%$ & $\mathrm{~N}$ & $\%$ & $\mathrm{n}$ & $\%$ & $\mathrm{n}$ & $\%$ \\
\hline $\begin{array}{l}\text { Acute cerebrovascular } \\
\text { accident }\end{array}$ & 8 & $12,1 \%$ & & & 3 & $10,0 \%$ & 2 & $20,0 \%$ \\
\hline Ischemic heart disease & 1 & $1,5 \%$ & 1 & $4,8 \%$ & & & & \\
\hline Diabetes mellitus type 2 & 1 & $1,5 \%$ & & & & & & \\
\hline Other causes & 4 & $6,1 \%$ & & & 1 & $3,3 \%$ & & \\
\hline Total & 14 & $21,2 \%$ & 1 & $4,8 \%$ & 4 & $13,3 \%$ & 2 & $20,0 \%$ \\
\hline &
\end{tabular}

Thus, analyzing the results by deaths with depending the gene polymorphism combinations of beta 1 and beta 3 adrenergic receptors, we can say that people the gene polymorphism combinations of beta 1 and beta 3 adrenergic receptors Arg/Arg+Trg/Trg were founded more frequently, than other combinations. The proportion of deaths less met with a combination of polymorphisms beta 1 and beta 3 adrenergic receptors Arg/Arg+Trg/Arg. Based on the data obtained by us can be divided into combination of polymorphisms of beta 1 and beta 3 adrenergic receptors on the «favorable» (Arg/Arg+Trg/Arg) and «non-favorable» (Arg/Arg+Trg/Trg, Arg/Gly+Trg/Trg, Arg/Gly+Trg/Arg) combinations of polymorphisms of genes. With «non-favorable» combinations of polymorphisms genes beta 1 and beta 3 adrenergic receptors most frequent cause of death was acute cerebrovascular accident (CVA).

Studying in alive patients anthropometric and biochemic parameters, we identified key differences by genes polymorphism. It was examined average clinical metabolic figures in the contrast with the 2003 year's parameters by the genes polymorphism combinations of beta 1 and beta 3 adrenergic receptors. Looking each combinations of the genes polymorphism combinations of beta 1 and beta 3 adrenergic receptors individually we took statistically significant changes of such determinants as BMI, $\mathrm{WC}$, triglycerides and glucose values in people with «non-favorable» combinations Arg/Arg+Trg/Trg (Table 3). But there were no statistically significantly differences in patients with genes polymorphism combinations of beta 1 and beta 3 adrenergic receptors Arg/Arg+Trg/Arg (Table 4) and Arg/Gly+Trg/Trg (Table 5). Volunteers with the genes polymorphism combinations of beta 1 and beta 3 adrenergic receptors Arg/Gly+Trg/Arg (Table 6) and Arg/Arg+Trg/Trg had statistically significant $\mathrm{p}$-value changes in BMI and WC.

Table 3

12-year developing metabolic risk factors depending on the genes polymorphism combinations of beta 1- and beta 3 adrenergic receptors Arg/Arg+Trg/Trg.

\begin{tabular}{|l|c|c|c|}
\hline \multirow{2}{*}{ Indicators } & \multicolumn{2}{|c|}{ Arg/Arg+Trg/Trg } & \multirow{2}{*}{ M } \\
\cline { 2 - 3 } & 2003 & 2015 & \\
\hline BMI, $\mathrm{kg} / \mathrm{m}^{2}$ & $26,29 \pm 5,68$ & $29,3 \pm 5,65$ & 0,002 \\
\hline WC, $\mathrm{m}$ & $87,84 \pm 13,64$ & $95,22 \pm 14,33$ & 0,001 \\
\hline total cholesterol, mmol/l & $5,19 \pm 0,96$ & $5,33 \pm 1,07$ & 0,296 \\
\hline HDL, $\mathrm{mmol} / \mathrm{l}$ & $1,2 \pm 0,31$ & $1,33 \pm 0,35$ & 0,032 \\
\hline Triglycerides, $\mathrm{mmol} / \mathrm{l}$ & $1,46 \pm 0,43$ & $1,73 \pm 0,96$ & 0,043 \\
\hline LDL, $\mathrm{mmol} / \mathrm{l}$ & $3,34 \pm 0,97$ & $3,25 \pm 0,77$ & 0,586 \\
\hline Glucose, $\mathrm{mmol} / \mathrm{l}$ & $5,61 \pm 2,07$ & $6,43 \pm 3,01$ & 0,000 \\
\hline Systolic blood pressure, $\mathrm{mmHg}$ & $132,5 \pm 25,52$ & $133,5 \pm 23,86$ & 0,838 \\
\hline Diastolic blood pressure, $\mathrm{mmHg}$ & $84,5 \pm 12,13$ & $84,2 \pm 11,57$ & 1,000 \\
\hline
\end{tabular}




\begin{tabular}{|c|c|c|c|c|c|c|}
\hline Impact Factor: & $\begin{array}{l}\text { ISRA (India) } \\
\text { ISI (Dubai, UAE } \\
\text { GIF (Australia) } \\
\text { JIF }\end{array}$ & $\begin{array}{l}=1.344 \\
=0.829 \\
=0.564 \\
=1.500\end{array}$ & $\begin{array}{l}\text { SIS (USA) } \\
\text { PИНЦ (Russia) } \\
\text { ESJI (KZ) } \\
\text { SJIF (Morocco) }\end{array}$ & $\begin{array}{l}=0.912 \\
=0.179 \\
=1.042 \\
=\mathbf{2 . 0 3 1}\end{array}$ & $\begin{array}{l}\text { ICV (Poland) } \\
\text { PIF (India) } \\
\text { IBI (India) }\end{array}$ & $\begin{array}{l}=6.630 \\
=1.940 \\
=4.260\end{array}$ \\
\hline
\end{tabular}

12-year developing metabolic risk factors depending on the genes polymorphism combinations of beta 1- and beta 3 adrenergic receptors Arg/Arg+Trg/Arg.

\begin{tabular}{|l|c|c|c|}
\hline \multirow{2}{*}{ Indicators } & \multicolumn{2}{|c|}{ Arg/ Arg+Trg/ Arg } & \multirow{2}{*}{ M(SD) } \\
\cline { 2 - 3 } & 2003 & 2015 & \\
\hline BMI, $\mathrm{kg} / \mathrm{m}^{2}$ & $27,31 \pm 5,00$ & $29,2 \pm 6,07$ & 0,282 \\
\hline WC, $\mathrm{m}$ & $89,77 \pm 14,74$ & $95,11 \pm 9,51$ & 0,176 \\
\hline total cholesterol, mmol/l & $5,14 \pm 0,8$ & $5,69 \pm 1,1$ & 0,074 \\
\hline HDL, mmol/l & $1,18 \pm 0,22$ & $1,29 \pm 0,38$ & 0,261 \\
\hline Triglycerides, $\mathrm{mmol} / \mathrm{l}$ & $1,55 \pm 0,52$ & $1,5 \pm 0,52$ & 0,760 \\
\hline LDL, $\mathrm{mmol} / \mathrm{l}$ & $3,26 \pm 0,78$ & $3,62 \pm 1,27$ & 0,276 \\
\hline Glucose, $\mathrm{mmol} / \mathrm{l}$ & $5,78 \pm 1,85$ & $5,77 \pm 1,22$ & 0,984 \\
\hline Systolic blood pressure, $\mathrm{mmHg}$ & $124,7 \pm 23,54$ & $128,61 \pm 24,6$ & 0,586 \\
\hline Diastolic blood pressure, $\mathrm{mmHg}$ & $81,66 \pm 12,83$ & $83,88 \pm 9,78$ & 0,551 \\
\hline
\end{tabular}

Table 5

12-year developing metabolic risk factors depending on the genes polymorphism combinations of beta 1- and beta 3 adrenergic receptors Arg/Gly+Trg/Trg.

\begin{tabular}{|l|c|c|c|}
\hline \multirow{2}{*}{ Indicators } & \multicolumn{2}{|c|}{ Arg/Gly+Trg/Trg } & \multirow{2}{*}{ M(SD) } \\
\cline { 2 - 3 } & 2003 & 2015 & \\
\hline BMI, $\mathrm{kg} / \mathrm{m}$ & $27,73 \pm 7,13$ & $29,51 \pm 6,032$ & 0,260 \\
\hline WC, $\mathrm{m}$ & $95,21 \pm 18,77$ & $98,04 \pm 13,28$ & 0,506 \\
\hline total cholesterol, mmol/l & $5,17 \pm 1,1$ & $5,35 \pm 0,88$ & 0,361 \\
\hline HDL, mmol/l & $1,2 \pm 0,32$ & $1,29 \pm 0,41$ & 0,304 \\
\hline Triglycerides, $\mathrm{mmol} / \mathrm{l}$ & $1,59 \pm 0,41$ & $1,65 \pm 0,6$ & 0,660 \\
\hline LDL, $\mathrm{mmol} / \mathrm{l}$ & $3,25 \pm 1,31$ & $3,22 \pm 0,79$ & 0,919 \\
\hline Glucose, mmol/l & $7,16 \pm 5,14$ & $7,03 \pm 3,25$ & 0,917 \\
\hline Systolic blood pressure, $\mathrm{mmHg}$ & $136,08 \pm 20,39$ & $136,30 \pm 27,22$ & 0,947 \\
\hline Diastolic blood pressure, $\mathrm{mmHg}$ & $87,82 \pm 12,77$ & $86,08 \pm 13,39$ & 0,406 \\
\hline
\end{tabular}

12-year developing metabolic risk factors depending on the genes polymorphism combinations of beta 1- and beta 3 adrenergic receptors Arg/Gly+Trg/Arg.

\begin{tabular}{|l|c|c|c|}
\hline \multirow{2}{*}{ Indicators } & \multicolumn{2}{|c|}{ Arg/Gly+Trg/Arg } & \multirow{2}{*}{ M(SD) } \\
\cline { 2 - 3 } & 2003 & 2015 \\
\hline BMI, $\mathrm{kg} / \mathrm{m}^{2}$ & $26,12 \pm 4,28$ & $29,41 \pm 2,14$ & 0,05 \\
\hline WC, $\mathrm{m}$ & $91,12 \pm 8,67$ & $97,62 \pm 8,76$ & 0,007 \\
\hline total cholesterol, mmol/l & $5,35 \pm 0,73$ & $5,03 \pm 0,9$ & 0,416 \\
\hline HDL, mmol/l & $1,1 \pm 0,21$ & $1,03 \pm 0,24$ & 0,519 \\
\hline Triglycerides, $\mathrm{mmol} / \mathrm{l}$ & $1,62 \pm 0,63$ & $2,07 \pm 0,99$ & 0,257 \\
\hline LDL, $\mathrm{mmol} / \mathrm{l}$ & $3,51 \pm 0,75$ & $2,86 \pm 0,64$ & 0,069 \\
\hline Glucose, mmol/l & $5,6 \pm 2,87$ & $6,25 \pm 1,77$ & 0,584 \\
\hline Systolic blood pressure, $\mathrm{mmHg}$ & $121,25 \pm 13,56$ & $123,75 \pm 7,3$ & 0,701 \\
\hline Diastolic blood pressure, $\mathrm{mmHg}$ & $77,5 \pm 7,07$ & $78,75 \pm 12,46$ & 0,791 \\
\hline
\end{tabular}

There were statistically significant $p$-value changes in BMI and WC in people with HVD and diabetes, depending on genes polymorphism combinations of beta 1 and beta 3 adrenergic receptors $\mathrm{Arg} / \mathrm{Arg}+\mathrm{Trg} / \mathrm{Trg}$ and $\mathrm{Arg} / \mathrm{Gly}+\mathrm{Trg} / \mathrm{Arg}$ (Table 7). 


\begin{tabular}{|c|c|c|c|c|c|c|}
\hline Impact Factor: & $\begin{array}{l}\text { ISRA (India) } \\
\text { ISI (Dubai, UAE } \\
\text { GIF (Australia) } \\
\text { JIF }\end{array}$ & $\begin{array}{l}=1.344 \\
=0.829 \\
=0.564 \\
=1.500\end{array}$ & $\begin{array}{l}\text { SIS (USA) } \\
\text { PИНЦ (Russia) } \\
\text { ESJI (KZ) } \\
\text { SJIF (Morocco) }\end{array}$ & $\begin{array}{l}=0.912 \\
=0.179 \\
=1.042 \\
=2.031\end{array}$ & $\begin{array}{l}\text { ICV (Poland) } \\
\text { PIF (India) } \\
\text { IBI (India) }\end{array}$ & $\begin{array}{l}=6.630 \\
=1.940 \\
=4.260\end{array}$ \\
\hline
\end{tabular}

Table 7

Average values of WC and BMI in people with HVD and diabetes mellitus type 2 by the genes polymorphism combinations of beta 1- and beta 3 adrenergic receptors

\begin{tabular}{|c|c|c|c|c|c|c|c|c|}
\hline $\begin{array}{l}\text { genes } \\
\text { polymorphis } \\
\mathrm{m} \\
\text { combinations } \\
\text { of beta } 1 \text { and } \\
\text { beta } \quad 3 \\
\text { adrenergic } \\
\text { receptors }\end{array}$ & \multicolumn{2}{|c|}{ Arg/Arg+Trg/Trg } & \multicolumn{2}{|c|}{ Arg/Arg+Trg/Arg } & \multicolumn{2}{|c|}{ Arg/Gly+Trg/Trg } & \multicolumn{2}{|c|}{ Arg/Gly+Trg/Arg } \\
\hline Indicators & 2003 & 2015 & 2003 & 2015 & 2003 & 2015 & 2003 & 2015 \\
\hline \multirow[t]{2}{*}{ WC } & $\begin{array}{l}91,8 \pm 15, \\
3\end{array}$ & $\begin{array}{l}112,0 \pm 9, \\
2\end{array}$ & $\begin{array}{l}99,7 \pm 6, \\
4\end{array}$ & $\begin{array}{l}101,5 \pm 12 \\
2\end{array}$ & $\begin{array}{l}101,2 \pm 16 \\
1\end{array}$ & $\begin{array}{l}102,8 \pm 15 \text {, } \\
9\end{array}$ & $\begin{array}{l}95,4 \pm 5, \\
9\end{array}$ & $\begin{array}{l}103,2 \pm 5, \\
1\end{array}$ \\
\hline & \multicolumn{2}{|c|}{$\mathrm{p}=0,014$} & \multicolumn{2}{|c|}{$p=0,634$} & \multicolumn{2}{|c|}{$\mathrm{p}=0,410$} & \multicolumn{2}{|c|}{$\mathrm{p}=0,0015$} \\
\hline \multirow[t]{2}{*}{ BMI } & $27,9 \pm 7,1$ & $35,6 \pm 3,1$ & $\begin{array}{l}29,9 \pm 1, \\
0\end{array}$ & $30,9 \pm 2,7$ & $30,4 \pm 5,7$ & $30,9 \pm 7,6$ & $\begin{array}{l}28,9 \pm 3, \\
6\end{array}$ & $32,1 \pm 6,3$ \\
\hline & \multicolumn{2}{|c|}{$p=0,014$} & \multicolumn{2}{|c|}{$p=0,475$} & \multicolumn{2}{|c|}{$p=0,935$} & \multicolumn{2}{|c|}{$\mathrm{p}=0,353$} \\
\hline
\end{tabular}

\section{CONCLUSION:}

The genes polymorphism combinations of beta 1 and beta 3 adrenergic receptors acts in HVD and diabetes mellitus type 2 [11].

The genes polymorphism combinations of beta 1 and beta 3 adrenergic receptors were divided into two groups: "favourable" and "non-favourable".

The genes polymorphism combinations of beta 1 and beta 3 adrenergic receptors Arg/Arg+Trg/Trg were founded more frequently, than other combinations. The least proportion of deaths were people with the genes polymorphism combinations of beta 1 and beta 3 adrenergic receptors Arg/Arg+Trg/Arg and the most proportion contained people with the gene polymorphism combinations of beta 1 and beta 3 adrenergic receptors
Arg/Arg+Trg/Trg. Among all causes of death acute stroke statistically significant founds more frequently than others.

"Non-favourable" genes polymorphism combinations of beta 1 and beta 3 adrenergic receptors were Arg/Arg+Trg/Trg and $\mathrm{Arg} / \mathrm{Gly}+\operatorname{Trg} / \mathrm{Arg}$, because of their predictors such classic factors as obesity, hyperglycemia, hypertriglyceridemia, which raise the risk of HVD and diabetes mellitus type 2. In patients with HVD and diabetes mellitus type 2 were determined changes with the gene polymorphism combinations of beta 1 and beta 3 adrenergic receptors Arg/Arg+Trg/Trg и Arg/Gly + Trg/Arg.

\section{References:}

1. Arner P (2000) Hunting for human obesity genes? Look in the adipose tissue! Int J Obes Relat Metab Disord. 2000 Nov;24 Suppl 4: 57-62.

2. Pulkkinen A1, Kareinen A, Saarinen L, Heikkinen S, Lehto S, Laakso M (1999) The codon 64 polymorphism of the beta3adrenergic receptor gene is not associat.ed with coronary heart disease or insulin resistance in nondiabetic subjects and non-insulin-dependent diabetic patients. Metabolism. 1999 Jul;48(7): 853

3. Baba T, Nakajima S, Yajima Y (1998) Beta3adrenergic receptor gene polymorphism is not associated with hypertension in NIDDM patients without nephropathy. Horm Metab Res. 1998 Oct;30(10):629-32.

4. Wang L1, Lu L, Zhang F, Chen Q, Shen W (2010) Polymorphisms of beta-adrenoceptor and natriuretic peptide receptor genes influence the susceptibility to and the severity of 


\begin{tabular}{l|lrl|l|ll} 
& ISRA (India) & $=\mathbf{1 . 3 4 4}$ & SIS (USA) & $=\mathbf{0 . 9 1 2}$ & ICV (Poland) & $=\mathbf{6 . 6 3 0}$ \\
Impact Factor: & ISI (Dubai, UAE) $=\mathbf{0 . 8 2 9}$ & PUHL (Russia) $=\mathbf{0 . 1 7 9}$ & PIF (India) & $=\mathbf{1 . 9 4 0}$ \\
& GIF (Australia) & $\mathbf{0 . 5 6 4}$ & ESJI (KZ) & $=\mathbf{1 . 0 4 2}$ & IBI (India) & $=\mathbf{4 . 2 6 0}$ \\
& JIF & $\mathbf{1 . 5 0 0}$ & SJIF (Morocco) & $=\mathbf{2 . 0 3 1}$ & & \\
\hline
\end{tabular}

idiopathic dilated cardiomyopathy in a Chinese cohort. J Card Fail. 2010 Jan;16(1):36-44. doi:10.1016/j.cardfail.2009.08.003. Epub 2009 Sep 25.

5. Berkinbayev S, Rysuly M, Mussayev A, Blum $\mathrm{K}$, Baitasova N, Mussagaliyeva A, Dzhunusbekova G, Makhatov B, Mussayev A, Yeshmanova A, Lesbekova R, Marchuk Y, Azhibekova R, Oscar-Berman M, Kulmaganbetov M (2014) Apolipoprotein Gene Polymorphisms (APOB, APOC111, APOE) in the Development of Coronary HeartDisease in Ethnic Groups of Kazakhstan.. J Genet Syndr Gene Ther. 2014 Jan 24;5(2):216.

6. Askarova SS (2009) «Vliyanie Trp64Arg polimorfizma gena beta3-adrenoreceptora na risk razvitiya saharnogo diabeta 2 tipa u bolnih abdominalnim ojireniem kazahskoy nasionalnosti. // Sb. mat. II Sezda terapevtov Respublika Kazahstan// Terapevticheskiy vestnkc.- 2009.- №3.- T.23.- page. 284.

7. Nuskabaeva GO (2010) «Predictori remodilirovaniya miocarda LG u genshin kazashek pri AG s AO», Almaty 2010.

8. Shalkharov SS, Shalkharova ZS, Askarova SS, Mukhamedov RS, Gmirko E (2009) «Vliyanie Trp64Arg polimorfizma gena beta3adrenoreceptora na risk razvitiya serdechnococudistih zabolevaniy u lits $\mathrm{s}$ abdominalnim ojireniem»// Terapevticheskiy vestnkc.- 2009.№2.- T.22.- page. 27-28.

9. Moraga F, Troncoso R, Mellado R, Díaz-Araya G, Vukasovic JL, Greig D, Perez O, García L, Roldán J, Ocaranza MP, Jali J, Chiong M,
Castro P (2008) Interactions between beta1 and beta2 adrenergic receptor polymorphisms as risk factors for chronic heart failure. Rev Med Chil. 2008 Nov;136(11):1371-80.

10. Paczkowska A1, Szperl M, Małek $Ł$, Mazurkiewicz Ł, Skóra E, Grzybowski J, Roszczynko M, Bilińska Z, Tesson F, Ruzyłło W (2009) Polymorphisms of the beta-1 and beta-2 adrenergic receptors in Polish patients with idiopathic dilated cardiomyopathy. Kardiol Pol. 2009 Mar;67(3):235-41.

11. Burguete-Garcia AI, Martinez-Nava GA, Valladares-Salgado A, Bermudez Morales VH, Estrada-Velasco B, Wacher N, PeraltaRomero J, Garcia-Mena J, Parra E, Cruz M (2014) Association of $\beta 1$ and $\beta 3$ adrenergic receptors gene polymorphisms with insulin resistance and high lipid profiles related to type 2 diabetes and metabolic syndrome. Nutr Hosp. 2014 Jun 1;29(6):1327-34. doi: 10.3305/nh.2014.29.6.7367

12. Shalkharova ZS (2006) Epidemiology and clinical aspects of metabolic syndrome in the South Kazakhstan // Almaty 2006.

13. Askarova SS (2010) «Risk razvitiya serdechnococudistih zabolevaniy i saharnogo diabeta 2 tipa $i$ ih korrektsiya pri abdominalnim ojireniem»// Almaty 2010.

14. Friedewald WT, Levy RI, Fredrickson DS (1972) Estimation of the concentration of lowdensity lipoprotein cholesterol in plasma without use of preparative centrifuge. // Clin. Chem.- 1972.- V. 18.- pp. 499-502. 